\title{
CARACTERÍSTICAS MATERNAS ASOCIADAS AL DIAGNÓSTICO DE MACROSOMIA FETAL EN UN HOSPITAL III-1 DE LA CAPITAL DE PERÚ
}

\author{
MATERNAL CHARACTERISTICS ASSOCIATED WITH THE FETAL MACROSOMY DIAGNOSIS IN A HOSPITAL III-1 \\ OF THE CAPITAL OF PERU
}

Kelly Huacachi-Trejo ${ }^{1, a}$, Lucy E. Correa-López ${ }^{1, b}$

\begin{abstract}
RESUMEN
Objetivo: Identificar cuáles son las características maternas asociadas al diagnóstico de macrosomía fetal en el Hospital Sergio E. Bernales de enero a diciembre del 2018. Métodos: Se realizó un estudio de tipo observacional, analítico, retrospectivo, de casos y controles. La población estudiada fueron las gestantes con el diagnostico de macrosomía fetal atendidas en el servicio de ginecoobstetricia del Hospital Sergio E. Bernales enero a diciembre del 2018. A través de una ficha de recolección de datos, se extrajo la información de las historias clínicas, las cuales después fueron procesadas según el programa de IBM SPSS Statistics v25. Resultados: De 532 pacientes estudiados se obtuvieron 133 casos y 399 controles. La edad materna varía entre 14 y 45 años (edad media de 27,01). Se encontró asociación estadísticamente significativa entre macrosomía fetal y las siguientes variables: embarazo postérmino ( $O R=13,613 \mathrm{IC} 95 \% 2,901-63,891)$, diabetes gestacional (OR 5,7 IC95\%2,5 -12,7), ganancia de peso excesiva (OR 1,833 IC95\%1,154-2,911), sexo del recién nacido (OR 1,83 IC95\%1,2-2,7) y edad de la madre (OR 1,7 IC95\%1,0-2,9). Al realizar el análisis multivariado no se encontró asociación con las variables edad de la madre $(P=0,228, O R \quad 1,510$ IC95\%0,773- 2,950) e IMC ( $\mathrm{P}=0,331$, OR 0,740 IC95\%0,403-1,358), por lo que se consideraron variables confusoras. Conclusión: Las características maternas asociadas al diagnóstico de macrosomía fetal son parto postérmino, diabetes gestacional, ganancia de peso excesiva y sexo del recién nacido.
\end{abstract}

Palabras clave: Macrosomía fetal; Diabetes gestacional; Ganancia de peso durante el embarazo; Edad materna; Emnbarazo postermino (fuente: DeCS BIREME).

\begin{abstract}
Objective: To identify the maternal characteristics associated with the diagnosis of fetal macrosomia at Sergio E. Bernales Hospital from January to December 2018. Methods: An observational, analytical, retrospective, case-control study was carried out. The population studied was pregnant women with a diagnosis of fetal macrosomia treated in the gynecoobstetrics service of Sergio E. Bernales Hospital from January to December 2018. Through a data collection sheet, the information from the medical records was extracted; the data was then processed according to the IBM SPSS Statistics v25 program. Results: Of 532 patients studied, 133 cases and 399 controls were obtained. Maternal age varies between 14 and 45 years (average age of 27.01). A statistically significant association was found between fetal macrosomia and the following variables: post-term pregnancy $(\mathrm{OR}=13.61395 \% \mathrm{Cl} 2.901-63.891)$, gestational diabetes (OR 5.7 IC95\% 2.5 -12.7), excessive weight gain (OR $1.83395 \% \mathrm{Cl} 1.154-2,911)$, sex of the newborn (OR $1.8395 \%$ $\mathrm{Cl} 1.2-2.7$ ) and age of the mother (OR $1.795 \% \mathrm{Cl} 1.0-2.9$ ). When performing the multivariate analysis, no association was found with the variables age of the mother $(P=0.228, O R 1.51095 \% \mathrm{Cl} 0.773-2.950)$ and BMI ( $P=0.331$, OR $0.74095 \% \mathrm{Cl} 0.403-1.358$ ), so they were considered confusing variables. Conclusion: The maternal characteristics associated with the diagnosis of fetal macrosomia are post-term delivery, gestational diabetes, excessive weight gain and sex of the newborn.
\end{abstract}

Key words: Fetal macrosomia; Gestational diabetes; weight gain during pregnancy, maternal age, postnatal pregnancy (source: MeSH NLM).

${ }^{1}$ Instituto de Investigación en Ciencias Biomédicas (INICIB), Universidad Ricardo Palma, Lima-Perú.

a Interna del Hospital Sergio E. Bernales.

b Economista.

Citar como: Kelly Huacachi-Trejo. Características maternas asociadas al diagnóstico de macrosomia fetal en un hospital III-1 de la capital de Perú. Rev. Fac. Med. Hum. Enero 2020; 20(1):76-81. DOI 10.25176/RFMH.v20i1.2549 


\section{INTRODUCCIÓN}

La macrosomía fetal se define como un peso al nacer igual o mayor a $4000 \mathrm{~g}$ o, en algunos contextos, un peso mayor de $4500 \mathrm{~g}$; aunque las conductas clínicas deberían tomarse a partir de los $4000 \mathrm{~g}^{(1,2)}$. Se sabe que la macrosomía fetal está asociada con una serie de complicaciones maternas y perinatales, como infección, hemorragia posparto, parto prolongado, desgarros perineales de grado alto, parto por cesárea, accidentes anestésicos y eventos tromboembólicos ${ }^{(3)}$. Según el Colegio Americano de Obstetras y Ginecología (ACOG), los fetos macrosómicos tienen un mayor riesgo de asfixia perinatal, aspiración de meconio, fractura de clavícula, lesión del plexo braquial y distocia de hombros ${ }^{(4)}$.

Aunque su prevalencia varía entre diferentes razas y diferentes grupos étnicos, afecta aproximadamente entre el 6 y $10 \%$ de todos los recién nacidos ${ }^{(1,3)}$. Un trabajo hecho por la Organización Mundial de la Salud en el año 2014 y 2015 reporta que en la región de Sudamérica, 7,6\% de todos los neonatos nacieron macrosómicos ${ }^{(5)}$. Asimismo, un estudio publicado el 2017 en el Perú encontró que la prevalencia global de macrosomía fue de 5,3\%; el cual es un porcentaje relativamente menor al encontrado a nivel mundial, sin embargo, lleva consigo muchas condiciones mórbidas ya explicadas previamente ${ }^{(6)}$.

Se sabe que la insulina materna es la principal hormona responsable del crecimiento fetal intrauterino. Durante el embarazo, la irregularidad de los niveles de glucosa en la sangre posprandial materna y la secreción excesiva de insulina, especialmente en el segundo y tercer trimestre, pueden causar macrosomía fetal ${ }^{(7)}$. EI estudio de hiperglucemia y resultados adversos del embarazo (HAPO) identificó una relación continua entre la glucosa materna y el aumento de peso al nacer ${ }^{(8)}$. Una revisión sistemática por Falavigne et al. ${ }^{(9)}$ informó que el tratamiento de la diabetes mellitus gestacional (DMG) fue eficaz para reducir las tasas de macrosomía, preeclampsia y distocia de hombros. Por lo tanto, el riesgo de macrosomía fetal debe considerarse durante la atención prenatal para mujeres embarazadas con diabetes mellitus pregestacional o gestacional. Así también, existen otros factores que han demostrado estar relacionados con la incidencia de macrosomía fetal, como lo es el perfil lipídico, principalmente los triglicéridos y los niveles de colesterol $\mathrm{HDL}^{(10,11)}$. Así como también la obesidad materna, que está relacionada con un mayor peso al nacimiento. Sin embargo, la mayoría de estudios que exploran estas relaciones fueron hechos en otros países distintos al nuestro, haciendo falta explorar estas relaciones en poblaciones como la nuestra. Es por ello que el objetivo de este estudio fue identificar cuáles son las características maternas asociadas al diagnóstico de macrosomía fetal en el Hospital Sergio E. Bernales (HNSEB) de enero a diciembre del 2018.

\section{MÉTODOS}

Se realizó un estudio observacional, analítico, retrospectivo, de casos y controles. La población estuvo conformada por puérperas con recién nacidos macrosómicos. Para el tamaño muestral se consideró una fórmula para estudios de casos y controles con un nivel de confianza de $95 \%$, poder estadístico de $80 \%$ y odds ratio igual a 2,02 . El tamaño de la muestra estuvo conformado por 133 casos (puérperas con recién nacidos macrosómicos) y 399 controles (puérperas con recién nacidos no macrosómicos). Se incluyeron a las puérperas cuyos controles médicos durante la gestación y atención del parto se realizaron en el Hospital Sergio E. Bernales durante el periodo de estudio. Los criterios de exclusión fueron tener historias clínicas con letra ilegible e información incompleta.

Se plantearon las siguientes variables: la edad de la madre, sexo del hijo, diabetes gestacional, diabetes pregestacional, embarazo postérmino. Para recolectar la información se solicitó a la Oficina de Apoyo a la Docencia e Investigación del Hospital Sergio Bernales, la autorización correspondiente, posteriormente se coordinó con la Oficina de Estadística para poder tener acceso a las historias clínicas.

Los números de historias clínicas se localizaron en el libro de registro de hospitalización del servicio de ginecología del Hospital Sergio E. Bernales en el año 2018. Posteriormente, se creó una base de datos electrónica, en el programa Microsoft Excel 2016, para seleccionarnos según técnica de muestreo. La técnica de recolección de datos fue la documentación. Se diseñó una ficha de datos para la recolección.

Se tomó una muestra de 133 casos de recién nacidos con macrosomía en una población 4363 puérperas de enero a diciembre del 2018 en el hospital Sergio Bernales. En este estudio se incluyeron la totalidad de los pacientes, los cuales cumplieron con los criterios de inclusión y exclusión.

Para buscar la asociación entre variables se hallaron los OR con sus respectivos intervalos de confianza al $95 \%$, usando regresión logística, se consideró un valor $p$ como significativo si es que este fue menor a 0,05 . El 
proyecto de investigación obtuvo la autorización del hospital nacional Sergio E. Bernales y de la Universidad Ricardo Palma para su realización.

\section{RESULTADOS}

Del total de la muestra evaluada, se observó que en relación a la variable edad, su media fue de 27,01 años con una desviación estándar de 6,74 , con un predominio del grupo etario en los pacientes menores de 35 años de edad con un $83,3 \%$. Además, se puede observar que las gestantes multíparas fueron las que predominaron con $64,8 \%$ del total. Con respecto al sexo del recién nacido, predominó el sexo femenino

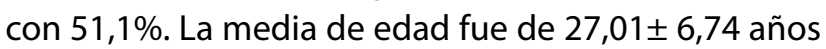
y el IMC promedio fue de $26,23 \pm 4,52 \mathrm{Kg} / \mathrm{m} 2$. La siguiente variable estudiada fue la edad gestacional, donde predomino el grupo de gestantes a término con un 90,0 \%, además se puede observar que en la variable culminación de gestación, predomino la cesárea con un $50,2 \%$. Esta y otras características se pueden observar en la tabla 1.

Tabla 1. Análisis univariado de las características maternas neonatal en pacientes del servicio de obstetricia del HNSEB.

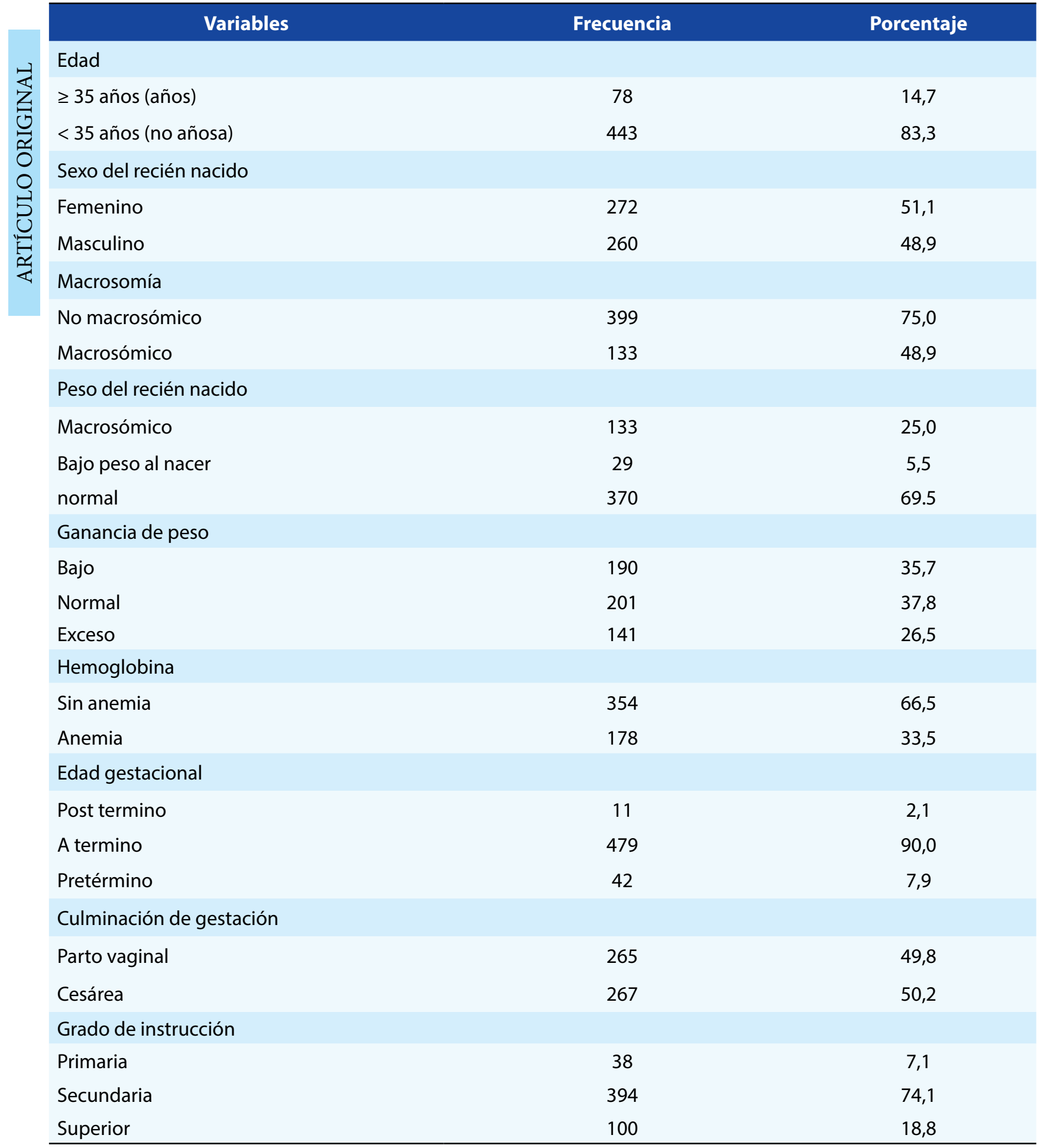


De acuerdo con el análisis bivariado, los factores de riesgo asociado a macrosomía fetal fueron edad de la madre, el sexo del recién nacido, la ganancia de peso baja, la ganancia de peso excesiva, embarazo post termino, diabetes pregestacional y diabetes gestacional. Los respectivos OR y valores de $p$ se pueden ver en la tabla 2.

Tabla 2. Análisis bivariado de los factores de riesgo para macrosomía en pacientes del servicio de obstetricia del HNSEB.

\begin{tabular}{|c|c|c|c|c|c|c|c|}
\hline \multirow{3}{*}{ Variable } & \multicolumn{4}{|c|}{ Macrosomía } & \multirow{3}{*}{ OR } & \multirow{3}{*}{ IC 95\% } & \multirow{3}{*}{ Valor de p } \\
\hline & \multicolumn{2}{|c|}{ Si } & \multicolumn{2}{|c|}{ No } & & & \\
\hline & $N=133$ & $\%$ & $N=399$ & $\%$ & & & \\
\hline Gestante añosa ( $\geq 35$ años) & 27 & 20,3 & 51 & 12,8 & 1,7 & $1,0-2,9$ & 0,034 \\
\hline $\begin{array}{l}\text { Sexo del recién nacido } \\
\text { masculino }\end{array}$ & 80 & 60,2 & 180 & 45,1 & 1,83 & $1,2-2,7$ & 0,003 \\
\hline Ganancia de peso excesiva* & 55 & 51,4 & 86 & 36,6 & 1,833 & $1,154-2,911$ & 0,010 \\
\hline Embarazo Postérmino & 9 & 7,0 & 2 & 0,6 & 13,613 & $2,901-63,891$ & $<0,001$ \\
\hline Diabetes pregestacional & 10 & 7,5 & 15 & 3,8 & 2,081 & $0,912-4,752$ & 0,076 \\
\hline Diabetes gestacional & 17 & 12,8 & 10 & 2,5 & 5,7 & $2,5-12,7$ & $<0,001$ \\
\hline $\mathrm{IMC} \geq 25 \mathrm{Kg} / \mathrm{m} 2$ & & & & & & & \\
\hline
\end{tabular}

*El grupo comparación fueron las madres con ganancia de peso normal.

En la tabla 3 se puede observar que, al realizar el análisis multivariado, se obtiene que las variables de sexo del recién nacido, ganancia de peso excesiva, embarazo postérmino y diabetes gestacional fueron las que tuvieron una asociación estadísticamente significativa, mientras que la edad de la madre e IMC en exceso fueron no estadísticamente significativas.

Tabla 3. Análisis multivariados de los factores maternos asociados a macrosomía en pacientes del servicio de obstetricia del HNSEB.

\begin{tabular}{lccc}
\hline \multicolumn{1}{c}{ Variable } & OR & IC 95\% & P Valor \\
\hline Gestante añosa ( $\geq 35$ años) & 1,510 & $0,773-2,950$ & 0,228 \\
Sexo del Recién Nacido & 1,822 & $1,082-3,067$ & 0,024 \\
Ganancia de Peso excesiva & 1,871 & $1,104-3,171$ & 0,020 \\
Embarazo postérmino & 16,043 & $1,795-143,377$ & 0,013 \\
Diabetes gestacional & 7,620 & $2,506-23,171$ & $<0,001$ \\
IMC mayor o igual a $25 \mathrm{Kg} / \mathrm{m} 2$ & 0,740 & $0,403-1,358$ & 0,331 \\
\hline
\end{tabular}

\section{DISCUSIÓN}

La macrosomía es una complicación obstétrica. Los informes anteriores han demostrado que los recién nacidos macrosómicos tienen un mayor riesgo de desarrollar hipertensión, obesidad y diabetes mellitus tipo 2 en la edad adulta ${ }^{(5)}$. En un estudio, realizado por Ismael, en Ica en el año 2016 encontró una prevalencia de $5 \%$ en macrosomía fetal ${ }^{(12)}$. En el año 2016, en
México reportaron una incidencia de $5,4 \%{ }^{(13)}$. Mientras que Quiroz ${ }^{(14)}$, en nuestro país, en el hospital María Auxiliadora, encontró una incidencia de $9,83 \%$ en ese mismo año. Valores similares a lo encontrado en el presente estudio.

La edad materna mayor a 35 años en mujeres que tuvieron un hijo o hija con macrosomía fetal tuvo cerca del doble de riesgo de desarrollar macrosomía 
fetal, resultados estadísticamente significativos sólo en el análisis bivariado, mas no en el multivariado. En un estudio realizado en Turquía, reportaron que las mujeres con edad mayor a 35 años tenían 1,5 veces más riesgo de desarrollar macrosomía fetal, siendo también significativo; en ambos estudios los valores son muy similares ${ }^{(15)}$.

En un estudio realizado por Córdova con un diseño de caso y controles realizado en el Centro Médico Naval el año 2017 encontró que el 63\% de los recién nacidos macrosómicos fueron de sexo masculino, con un OR de 2,02 y un valor $\mathrm{p}=0,027^{(16)}$; para nosotros, el recién nacido de sexo masculino tuvo un OR similar, ligeramente mejor, siendo su relación con macrosomía fetal estadísticamente significativa, lo que nos indica que el género masculino actúa como un factor de riesgo para la presentación de macrosomía fetal. La ganancia de peso excesiva estuvo en más de la mitad de mujeres con un hijo que tuvo macrosomía fetal, con un riesgo cercano al doble de desarrollar esta enfermedad; relación que se mantuvo al hacer el análisis multivariado. Estos resultados concuerdan con el estudio realizado en el hospital San José del año 2017 por Alva, en el que este factor tuvo un OR de 1,42 y un valor de $\mathrm{p}$ significativo, por lo cual sería un factor de riesgo para macrosomía(17).

La edad gestacional postérmino represento un factor de riesgo para macrosomia fetal, en el análisis bivariado y en el análisis multivariado. Este resultado es similar al encontrado en un estudio realizado por Leda en el país de Paraguay, donde se encontró que el embarazo postérmino presentó un OR de 14,7 veces más riego de desarrollar macrosomía con un $\mathrm{p}$ menor de 0,001 , siendo estadísticamente significativo ${ }^{(19)}$. Así también, un IMC en exceso fue un factor de riesgo para macrosomia fetal, que sin embargo no mantuvo esta asociación en el análisis multivariado. Resultado que contrasta lo encontrado en un estudio realizado en el servicio de neonatología del hospital San José en el 2017 por Alva, quien encontró que el IMC estuvo presente en $60,7 \%$ de los casos de macrosomía fetal y tuvo un OR 1,97 veces más riego de desarrollar macrosomía y con un valor de $\mathrm{p}$ estadísticamente significativo(20). En otro estudio realizado en el Hospital Vitarte realizado entre enero y julio del año 2018 por Arroyo se encontró un OR de 7,22 veces más riesgo de presentar macrosomía fetal, con un p estadísticamente

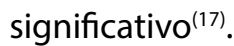

La presencia de diabetes pregestacional no represento un factor de riesgo de macrosomia fetal, resultado análogo al del estudio realizado en el Hospital Regional Guillermo Díaz de la Vega durante enero del 2016 y febrero del 2018 por Midward, que encontró un OR mayor a 1 y un $p=5,754$; corroborando que no existe correlaciones significativas, entre ésta patología materna y la macrosomía del recién nacido(20). Sin embargo, la presencia de diabetes gestacional si fue un factor de riesgo para macrosomía fetal, en el análisis bi y multivariado. Lo cual concuerda con lo encontrado en un estudio realizado en el Centro Médico Naval por Verastegui en el año 2014 encontrando un OR de 2,5 y un $\mathrm{p}=0,027$; siendo estadísticamente significativo. Esto podría explicarse debido a que los hijos de madres diabéticas sufren un efecto anabolizante debido al hiperinsulinismo fetal ${ }^{(8)}$.

Las limitaciones del presente estudio constituyen la no medición de las variables sociodemográficas y los valores de laboratorio como glucosa en ayunas, hiperinsulinemia o hipertrigliceridemia. Sin embargo, los resultados presentados permiten diseñar intervenciones para prevenir esta patología en nuestro medio.

\section{CONCLUSIÓN}

En la población estudiada, el sexo del recién nacido, la presencia de diabetes gestacional, la ganancia de peso excesiva y el embarazo postérmino fueron factores de riesgo de macrosomía en el neonato.

Contribuciones de autoría: El autor participó en la génesis de la idea, diseño de proyecto, recolección, análisis de la información y preparación del manuscrito del presente trabajo de investigación.

Financiamiento: Autofinanciado.

Conflicto de interés: El autor declara no tener conflicto de interés en la publicación de este artículo.

Recibido: 10 de octubre del 2019

Aprobado: 13 de diciembre del 2019

Correspondencia: Kelly Huacachi Trejo.

Dirección: INICIB, Facultad de Medicina Humana, Edificio I- 208. 2do piso. Avenida Benavides 5440, Surco, Lima-Perú.

Teléfono: 999115611

Correo: kelita_bth@hotmail.com 


\section{REFERENCIAS BIBLIOGRÁFICAS}

1. Amini $P$, Maroufizadeh S, Hamidi O, Samani RO, Sepidarkish M. Factors associated with macrosomia among singleton live-birth: A comparison between logistic regression, random forest and artificial neural network methods. Epidemiol Biostat Public Health [Internet]. 21 de diciembre de 2016 [citado 20 de julio de 2017];13(4): 1 - 9. Disponible en: http://ebph.it/article/ view/11985

2. Yamamoto M, Insunza A. Macrosomía fetal. Contacto Científico. 2016; 6(4): 262-6. Disponible en: http://contactocientifico.alemana.cl/ojs/index.php/cc/ article/view/385

3. Chávez-Atoche KV. Factores maternos asociados a macrosomía en recién nacidos de puérperas atendidas en el Hospital Nacional Sergio E. Bernales, Mayo-Octubre 2014. Rev Peru Obstet Enferm [Internet]. 21 de septiembre de 2014 [citado 9 de noviembre de 2018]; 10(2). Disponible en: https://www. aulavirtualusmp.pe/ojs/index.php/rpoe/article/view/725

4. Jaurigue-Arestegui $\mathrm{KC}$, Uría-Guerrero NM, Vargas-Huamantumba1 $Y^{\prime}$ Soberon UEM-. Los factores asociados a la gestante y al recién nacido macrosómico en el Hospital Regional de Ica. Rev Méd Panacea. 2014; 4(1) 17 - 21. Disponible en: file:///C:/Users/Onice/Downloads/157-Articulo\%20 Original-617-1-10-20190808.pdf

5. World Health Organization. World health statistics 2014. 2014. Disponible en: https://www.who.int/gho/publications/world_health_statistics/2014/en/

6. Ledo Alves da Cunha JA, Sobrino Toro M, Gutiérrez C, Alarcón-Villaverde J. Prevalencia y factores asociados a macrosomía en Perú, 2013. Rev Peru Med Exp Salud Publica. 2017; 34(1): 36-42. Disponible en: http://dx.doi. org/10.17843/rpmesp.2017.341.2765

7. Reyes RÁ, Pen MH, Cerda CIS, Ramírez RIC. Factores de riesgo del recién nacido macrosómico. Pediatría México. 2013; 15(1): 6-11. Disponible en: https://www. medigraphic.com/cgi-bin/new/resumen.cgi?IDARTICULO=40681

8. Córdova Verástegui R. factores de riesgo maternos asociados a la presentacion de recien nacidos macrosomicos en el centro médico naval cirujano mayor santiago tavara en el periodo julio 2014 a julio 2016. [Bachiller] Universidad Ricardo Palma; 2017. Disponible en: http://cybertesis.urp.edu.pe/ handle/urp/978

9. Gaudet L, Wen SW, Walker M. The combined effect of maternal obesity and fetal macrosomia on pregnancy outcomes. J Obstet Gynaecol. 2014;36(9):776 84. doi: 10.1016/S1701-2163(15)30479-5.

10. Herrera Martínez A, Palomares Ortega R, Bahamondes Opazo $\mathrm{R}$ Moreno-Moreno P, Molina Puerta MJ, Gálvez-Moreno MA. Hyperlipidemia during gestational diabetes and its relation with maternal and offspring complications. Nutr Hosp. 2018;35(3):698-706. doi: 10.20960/nh.1539.

11. Cruz J, Grandía R, Padilla L, Rodríguez S, Hernández P, Lang J, et al. Macrosomia Predictors in Infants Born to Cuban Mothers with Gestational
Diabetes. MEDICC Rev. 2015; 17(3): 27-32. Disponible en: https://www.ncbi. nlm.nih.gov/pubmed/26947155

12. Gonzáles-Tipiana IR, Macrosomía Fetal: Prevalencia, Factores de riesgo Asociados Y complicaciones en el hospital regional de lca. Rev. Méd. Panacea. 2012; 2(29: 55 - 7. Disponible en: file:///C:/Users/Onice/Downloads/129 Articulo\%20Original-509-1-10-20190808.pdf

13. García De la Torre Jl, Rodríguez-Valdez A, Delgado Rosas. Factores de riesgo de macrosomía fetal en pacientes sin diabetes mellitus gestacional. artículo original Gineco Obstet Mex. 2016; 84(3): 164-171. Disponible en: https://www. medigraphic.com/cgi-bin/new/resumen.cgi?IDARTICULO=71507

14. Quiroz Flores, RS. Factores de riesgo para macrosomía fetal en el Hospita María Auxiliadora: enero - diciembre 2016. Lima - Perú: Universidad Ricardo palma; 2018. Disponible en: http://webcache.googleusercontent.com/ search?q=cache:http://repositorio.urp.edu.pe/handle/URP/1228

15. Usta A, Sancakli usta C, Yildiz A. Frequency of fetal macrosomia and the associated risk factors in pregnancies without gestational diabetes mellitus . The Pan African Medical Journal. 2017; 26: 62. doi:10.11604/ pamj.2017.26.62.11440

16. Córdova Verástegui R, Gonzales-Mendes MJM, Correa-Lopez LE. Factores de riesgo materno asociados a la presentación de recién nacidos macrosómicos en el centro médico naval cirujano mayor Santiago Távara en el periodo julio 2014 a julio 2016. Rev. Fac. Med. Hum. 2017; 17(1): 48 - 55. doi: 10.25176/ RFMH.v17.n1.748

17. Alva Huarai RA. Factores asociados a macrosomía en el recién nacido en el servicio de neonatología del hospital san José del callao en el año 2017. Lima-Peru: Universidad nacional federico Villarreal; 2018. http://repositorio. unfv.edu.pe/handle/UNFV/1681?locale-attribute=en

18. Huaita franco ML. Factores de riesgo asociados a macrosomía fetal en el hospital uldarico rocca Fernández de villa el salvador, enero -julio 2015. Lima Perú:UniversidadSanMartinPorras;2015.Disponibleen:https://alicia.concytec. gob.pe/vufind/Record/USMP dee1ac5ef216edb236bffe713dcd6c8e

19.Romero Nardelli LB. Factores de riesgo asociados a la macrosomía fetal. Revista Nac. (Itauguá). 2014; 6(1): 16-24. Disponible en: http://scielo.iics.una. py/scielo.php?script=sci_abstract\&pid=S2072-81742014000100003\&lng=es \&nrm=iso\&tlng=es

20. Arroyo calderón, JJ. Condiciones materno-fetales asociadas a macrosomía fetal en gestantes sometidas a cesárea del Hospital de Vitarte, de enero a julio del 2018. Lima-Peru: Universidad Ricardo palma ; 2018. http://repositorio.urp. edu.pe/handle/URP/1858?show=full

21. Pizarro flores MF. Factores predictores de macrosomía fetal en el hospital regional Guillermo días de la vega enero 2016 - febrero 2018. Puno-Perú: Universidad nacional Altiplano; 2019. Disponible en: http://repositorio.unap. edu.pe/handle/UNAP/7712

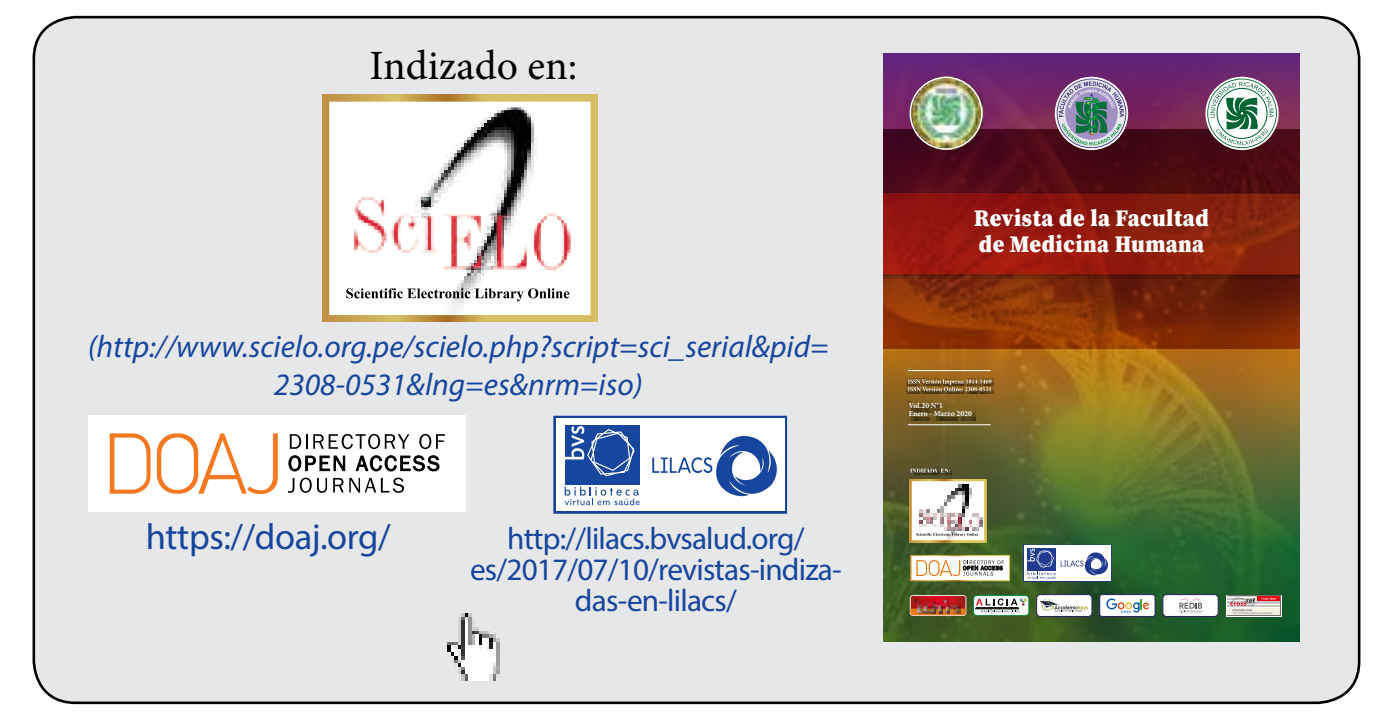

